\title{
Ammonia Promoted Fragmentation of
}

\section{2-Alkyl- and 2,4-Dialkyl-3-iodo-1-oxocyclohexan-2,4-carbolactones}

\author{
Mingshi Dai, Xuqing Zhang, Seock-Kyu Khim,* and Arthur G. Schultz \\ Department of Chemistry, Rensselaer Polytechnic Institute, Troy, New York 12180 \\ Supporting Information
}

\section{Table of Contents}

General Procedure for Fragmentation of $\mathbf{1}$ with $\mathrm{NH}_{3}$

Cyclization of $\mathbf{5 f}$

Hoffmann Rearrangement of $\mathbf{5 g}$

Cyclization of $\mathbf{1 6}$ in the Presence of TFA

Figure 1. ORTEP drawing of $\mathbf{1 5}$

Table 1. Crystal data and structure refinement for $\mathbf{1 5}$

Figure 2. Energy-minimized structures of $\mathbf{1 4}$ and $\mathbf{1 5 .}$

Figure 3. Energy-minimized structures of $\mathbf{1 8}$ and 19.

${ }^{1} \mathrm{H}$ and ${ }^{13} \mathrm{C}$ NMR spectra of $\mathbf{5 b}, \mathbf{1 4 + 1 5}, \mathbf{1 8}$, and 19

General Procedure for Fragmentation of $\mathbf{1}$ with $\mathbf{N H}_{3}$. To a solution of $\mathbf{1 a}(280 \mathrm{mg}, 1.0 \mathrm{mmol})$ in dry THF ( $5 \mathrm{~mL}$ ) was introduced $\mathrm{NH}_{3}$ (ca. $3 \mathrm{~mL}$ by condensing with cold-finger condenser) at $-78{ }^{\circ} \mathrm{C}$. The resulting solution was stirred for $0.5 \mathrm{~h}$ and slowly allowed to warm to room temperature under a stream of nitrogen until $\mathrm{NH}_{3}$ and THF were completely evaporated. ${ }^{1} \mathrm{H}$ NMR spectrum analysis of the crude mixture showed the ratio of $\mathbf{5 a}$ and $\mathbf{6 a}$ to be $76: 24$. The crude 
residue was partitioned between water and $\mathrm{CH}_{2} \mathrm{Cl}_{2}$. The organic layers were separated, dried over anhydrous $\mathrm{Na}_{2} \mathrm{SO}_{4}$, and concentrated under reduced pressure which was purified by flash column chromatography (hexanes/EtOAc, 4:1 to 1:1) to give 5a (99 mg, 59\%) and 6a (30 mg, 18\%). (5R)-[3-Methyl-5-(2'-aminocarbonyl)ethyl]furan-2(5H)-one (5a): white solid; $[\alpha]^{25}-121.4(c$ 2.52, MeOH); ${ }^{1} \mathrm{H}$ NMR (500 MHz, $\mathrm{CDCl}_{3}$ ) $\delta 7.05$ (s, $1 \mathrm{H}$ ), 5.85 (br s, $\left.1 \mathrm{H}\right), 5.69$ (br s, $\left.1 \mathrm{H}\right), 4.97$ (m, 1H), 2.43-2.32 (m, 2H), 2.26-2.20 (m, 1H), $1.90(\mathrm{~d}, J=1.5 \mathrm{~Hz}, 3 \mathrm{H}), 1.82-1.74(\mathrm{~m}, 1 \mathrm{H}) ;{ }^{13} \mathrm{C}$ NMR (125 MHz, $\left.\mathrm{CDCl}_{3}\right) \delta 173.9,164.4,148.7,130.1,80.1,30.4,28.6,10.6$; IR 3422, 3207, $17481669 \mathrm{~cm}^{-1}$; CIMS $170\left(\mathrm{M}^{+}+1,100\right)$. Anal. Calcd for $\mathrm{C}_{8} \mathrm{H}_{11} \mathrm{NO}_{3}: \mathrm{C}, 56.80 ; \mathrm{H}, 6.55 ; \mathrm{N}, 8.28$. Found: $\quad$ C,$\quad 56.72 ; \quad H, \quad 6.40 ; \quad$ N， 8.17. (2R,2E)-2-Methyl-3-[tetrahydro-5-oxofuranyl]-2propenamide (6a): white solid; $[\alpha]^{25}{ }_{\mathrm{D}}-72.6$ (c 1.15, $\left.\mathrm{MeOH}\right) ;{ }^{1} \mathrm{H}$ NMR $\left(500 \mathrm{MHz}, \mathrm{CD}_{3} \mathrm{OD}\right) \delta$ $6.36(\mathrm{~d}, J=8.0 \mathrm{~Hz}, 1 \mathrm{H}), 5.26(\mathrm{q}, J=7.6 \mathrm{~Hz}, 1 \mathrm{H}), 2.62-2.58(\mathrm{~m}, 2 \mathrm{H}), 2.51-2.45(\mathrm{~m}, 1 \mathrm{H}), 2.07-$ $1.99(\mathrm{~m}, 1 \mathrm{H}), 1.94(\mathrm{~s}, 3 \mathrm{H}) ;{ }^{13} \mathrm{C}$ NMR (125 MHz, $\left.\mathrm{CD}_{3} \mathrm{OD}\right) \delta 177.1,173.0,137.9,131.4,67.3$, 32.0, 31.1, 12.9; IR 3689, 3373, 1734, $1668 \mathrm{~cm}^{-1}$; CIMS $170\left(\mathrm{M}^{+}+1,100\right)$. Anal. Calcd for $\mathrm{C}_{8} \mathrm{H}_{11} \mathrm{NO}_{3}: \mathrm{C}, 56.80 ; \mathrm{H}, 6.55 ; \mathrm{N}, 8.28$. Found: C, 56.77; H, 6.49; N, 8.16.

(5R)-[3-(3'-Chloro)propyl-5-(2'-aminocarbonyl)ethyl]furan-2(5H)-one (5b): white solid; mp $101-102{ }^{\circ} \mathrm{C} ;[\alpha]^{26}{ }_{\mathrm{D}}-21.7(c 0.23, \mathrm{MeOH}) ;{ }^{1} \mathrm{H} \mathrm{NMR}\left(500 \mathrm{MHz}, \mathrm{CDCl}_{3}\right) \delta 7.10(\mathrm{~s}, 1 \mathrm{H}), 5.69$ (br $\mathrm{d}, J=60.5 \mathrm{~Hz}, 2 \mathrm{H}), 5.03(\mathrm{~m}, 1 \mathrm{H}), 3.55(\mathrm{t}, J=6.4 \mathrm{~Hz}, 2 \mathrm{H}), 2.46(\mathrm{t}, J=7.4 \mathrm{~Hz}, 2 \mathrm{H}), 2.43-2.33(\mathrm{~m}$, $2 \mathrm{H}), 2.28-2.22(\mathrm{~m}, 1 \mathrm{H}), 2.07-2.00(\mathrm{~m}, 2 \mathrm{H}), 1.85-1.77(\mathrm{~m}, 1 \mathrm{H}) ;{ }^{13} \mathrm{C} \mathrm{NMR}\left(125 \mathrm{MHz}, \mathrm{CDCl}_{3}\right) \delta$ $173.8,173.4,148.9,133.0,80.2,43.9,30.2,29.9,28.5,22.6$; IR 3386, 3180, 1738, $1653 \mathrm{~cm}^{-1}$; CIMS $234\left(\mathrm{M}^{+}+1,32\right), 232\left(\mathrm{M}^{+}+1,100\right)$; HRMS calcd for $\mathrm{C}_{10} \mathrm{H}_{14}{ }^{35} \mathrm{ClNO}_{3}\left(\mathrm{M}^{+}+1\right) 232.0740$, found 232.0744 . 
(5R)-[3-(3'-Benzyloxy)propyl-5-(2'-aminocarbonyl)ethyl]furan-2(5H)-one (5c): white solid; mp 75-77 ${ }^{\circ} \mathrm{C} ;[\alpha]^{25}-17.5\left(c\right.$ 1.2, MeOH); ${ }^{1} \mathrm{H}$ NMR (500 MHz, $\left.\mathrm{CDCl}_{3}\right) \delta 7.27(\mathrm{~m}, 2 \mathrm{H}), 7.24(\mathrm{~m}$, 2H), $7.20(\mathrm{~m}, 1 \mathrm{H}), 6.89(\mathrm{~s}, 1 \mathrm{H}), 6.04(\mathrm{~s}, 1 \mathrm{H}), 5.88(\mathrm{~s}, 1 \mathrm{H}), 4.86(\mathrm{~m}, 1 \mathrm{H}), 4.40(\mathrm{~s}, 2 \mathrm{H}), 3.41(\mathrm{t}, J=$ $5.4 \mathrm{~Hz}, 2 \mathrm{H}), 2.28(\mathrm{t}, J=7.1 \mathrm{~Hz}, 2 \mathrm{H}), 2.23(\mathrm{~m}, 2 \mathrm{H}), 2.09(\mathrm{~m}, 1 \mathrm{H}), 1.76(\mathrm{t}, J=6.9 \mathrm{~Hz}, 2 \mathrm{H}), 1.67$ $(\mathrm{m}, 1 \mathrm{H}) ;{ }^{13} \mathrm{C} \mathrm{NMR}\left(125 \mathrm{MHz}, \mathrm{CDCl}_{3}\right) \delta 174.2,173.6,148.1,138.1,133.8,128.2,127.8,127.5$, 127.4, 80.1, 72.7, 68.9, 30.0, 27.2, 21.9; IR 3348, 1754, $1666 \mathrm{~cm}^{-1}$; CIMS $304\left(\mathrm{M}^{+}+1,100\right)$. Anal. Calcd for $\mathrm{C}_{17} \mathrm{H}_{21} \mathrm{NO}_{4}$ : C, 67.33; H, 6.93. Found: C, 67.05; H, 6.93.

(5R)-[3-[(2'-Trimethylsilyl)ethyloxy]methyl-5-(2'-aminocarbonyl)ethyl]furan-2(5H)-one (5d): white solid; mp $83-85{ }^{\circ} \mathrm{C}$; $[\alpha]^{25}{ }_{\mathrm{D}}-15.3$ (c 5.10, MeOH); ${ }^{1} \mathrm{H}$ NMR $\left(500 \mathrm{MHz}, \mathrm{CDCl}_{3}\right) \delta$ $7.28(\mathrm{~s}, 1 \mathrm{H}), 6.35(\mathrm{~s}, 1 \mathrm{H}), 6.16(\mathrm{~s}, 1 \mathrm{H}), 5.02(\mathrm{~s}, 1 \mathrm{H}), 4.11(\mathrm{~s}, 2 \mathrm{H}), 3.54(\mathrm{t}, J=7.8 \mathrm{~Hz}, 2 \mathrm{H}), 2.34$ $(\mathrm{d}, J=4.9 \mathrm{~Hz}, 2 \mathrm{H}), 2.18(\mathrm{~d}, J=7.8 \mathrm{~Hz}, 1 \mathrm{H}), 1.75(\mathrm{~m}, 1 \mathrm{H}), 0.91(\mathrm{t}, J=7.8 \mathrm{~Hz}, 2 \mathrm{H}),-0.04(\mathrm{~s}, 9 \mathrm{H})$;

${ }^{13} \mathrm{C}$ NMR $\left(125 \mathrm{MHz}, \mathrm{CDCl}_{3}\right) \delta 174.3,172.1,149.8,131.8,80.9,68.5,63.5,30.3,28.4,17.8,-1.7$; IR 3350, 1756, $1668 \mathrm{~cm}^{-1}$; CIMS $287\left(\mathrm{M}^{+}+1,100\right)$. Anal. Calcd for $\mathrm{C}_{13} \mathrm{H}_{23} \mathrm{NO}_{4} \mathrm{Si}: \mathrm{C}, 54.74 ; \mathrm{H}$, 8.07. Found: C, 54.41; H, 8.11.

(5R)-[3-Methyl-5-methyl-5-(2'-aminocarbonyl)ethyl]furan-2(5H)-one (5e): white solid; mp $86-88{ }^{\circ} \mathrm{C} ;[\alpha]^{25}-37.5\left(c 0.8, \mathrm{CHCl}_{3}\right) ;{ }^{1} \mathrm{H}$ NMR $\left(500 \mathrm{MHz}, \mathrm{CDCl}_{3}\right) \delta 6.94$ (s, 1H), 5.93 (br s, 1H), $5.65(\mathrm{br} \mathrm{s}, 1 \mathrm{H}), 2.23-2.01(\mathrm{~m}, 4 \mathrm{H}), 1.86(\mathrm{~s}, 3 \mathrm{H}), 1.43(\mathrm{~s}, 3 \mathrm{H}) ;{ }^{13} \mathrm{C} \mathrm{NMR}\left(125 \mathrm{MHz}, \mathrm{CDCl}_{3}\right) \delta$ 174.6, 174.1, 153.1, 129.3, 86.1, 33.2, 29.6, 24.6, 10.6; IR 1746, $1667 \mathrm{~cm}^{-1}$; CIMS $184\left(\mathrm{M}^{+}+1\right.$, 100). Anal. Calcd for $\mathrm{C}_{9} \mathrm{H}_{13} \mathrm{NO}_{3}$ : C, 59.00; H, 7.15; N, 7.65. Found: C, 59.02; H, 7.07; N, 7.65.

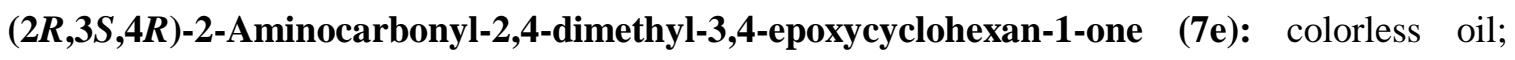
$[\alpha]^{25}{ }_{\mathrm{D}}-190.9\left(c\right.$ 1.1, $\left.\mathrm{CHCl}_{3}\right) ;{ }^{1} \mathrm{H}$ NMR $\left(500 \mathrm{MHz}, \mathrm{CDCl}_{3}\right) \delta 6.93$ (br s, $\left.1 \mathrm{H}\right), 5.61$ (br s, $\left.1 \mathrm{H}\right), 3.34$ $(\mathrm{s}, 1 \mathrm{H}), 2.61-2.10(\mathrm{~m}, 4 \mathrm{H}), 1.50(\mathrm{~s}, 3 \mathrm{H}), 1.43(\mathrm{~s}, 3 \mathrm{H}) ;{ }^{13} \mathrm{C} \mathrm{NMR}\left(125 \mathrm{MHz}, \mathrm{CDCl}_{3}\right) \delta 208.2$, 
171.9, 62.6, 58.0, 54.8, 34.8, 26.7, 22.1, 20.3; IR 1711, $1660 \mathrm{~cm}^{-1}$; CIMS $184\left(\mathrm{M}^{+}+1,100\right)$. Anal. Calcd for $\mathrm{C}_{9} \mathrm{H}_{13} \mathrm{NO}_{3}$ : C, 59.00; H, 7.15; N, 7.65. Found: C, 59.03; H, 7.18; N, 7.64. (5R)-[3-Ethyl-5-methyl-5-(2'-aminocarbonyl)ethyl]furan-2(5H)-one (5f): white solid; mp $75-78{ }^{\circ} \mathrm{C} ;[\alpha]^{25}-21.0\left(c\right.$ 1.0, $\left.\mathrm{CHCl}_{3}\right) ;{ }^{1} \mathrm{H} \mathrm{NMR}\left(500 \mathrm{MHz}, \mathrm{CDCl}_{3}\right) \delta 6.89$ (s, 1H), 6.15 (br s, 1H), 5.87 (br s, 1H), $2.20(\mathrm{~m}, 3 \mathrm{H}), 2.04(\mathrm{~m}, 3 \mathrm{H}), 1.40(\mathrm{~s}, 3 \mathrm{H}), 1.09$ (t, $J=7.5 \mathrm{~Hz}, 3 \mathrm{H}) ;{ }^{13} \mathrm{C} \mathrm{NMR}$ $\left(125 \mathrm{MHz}, \mathrm{CDCl}_{3}\right) \delta 174.8,173.6,151.5,135.3,86.2,33.2,29.5,24.6,18.6,11.8 ;$ IR 3350, 1746, $1667 \mathrm{~cm}^{-1}$; CIMS $198\left(\mathrm{M}^{+}+1,100\right)$. Anal. Calcd for $\mathrm{C}_{10} \mathrm{H}_{15} \mathrm{NO}_{3}: \mathrm{C}, 60.90 ; \mathrm{H}, 7.67 ; \mathrm{N}, 7.10$. Found: C, 61.07; H, 7.64; N, 7.19.

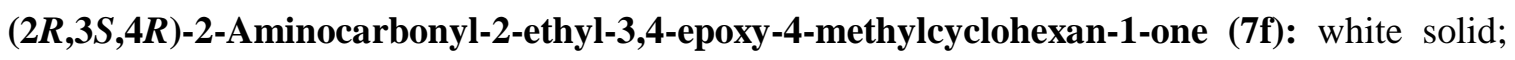
$[\alpha]^{25}{ }_{\mathrm{D}}-165.5\left(c 0.9, \mathrm{CHCl}_{3}\right) ;{ }^{1} \mathrm{H}$ NMR $\left(500 \mathrm{MHz}, \mathrm{CDCl}_{3}\right) \delta 6.83(\mathrm{br} \mathrm{s}, 1 \mathrm{H}), 6.05(\mathrm{br} \mathrm{s}, 1 \mathrm{H}), 3.36$ $(\mathrm{s}, 1 \mathrm{H}), 2.54(\mathrm{~m}, 1 \mathrm{H}), 2.29-2.03(\mathrm{~m}, 4 \mathrm{H}), 1.80(\mathrm{~m}, 1 \mathrm{H}), 1.51(\mathrm{~s}, 3 \mathrm{H}), 0.86(\mathrm{t}, J=7.4 \mathrm{~Hz}, 3 \mathrm{H}) ;{ }^{13} \mathrm{C}$ NMR $\left(125 \mathrm{MHz}, \mathrm{CDCl}_{3}\right) \delta 209.4,171.2,62.1,59.6,58.1,35.6,29.6,26.9,22.1,9.6 ;$ IR 3346, 3349, $1682 \mathrm{~cm}^{-1}$; CIMS $198\left(\mathrm{M}^{+}+1,100\right)$. Anal. Calcd for $\mathrm{C}_{10} \mathrm{H}_{15} \mathrm{NO}_{3}: \mathrm{C}, 60.90 ; \mathrm{H}, 7.67 ; \mathrm{N}$, 7.10. Found: C, 60.99; H, 7.51; N, 7.12.

(5R)-[3-Ethyl-5-benzyl-5-(2'-aminocarbonyl)ethyl]furan-2(5H)-one $(5 \mathrm{~g})$ : white solid; $\mathrm{mp}$ $112-115{ }^{\circ} \mathrm{C} ;[\alpha]^{25}+1.70\left(c\right.$ 1.2, $\left.\mathrm{CHCl}_{3}\right) ;{ }^{1} \mathrm{H}$ NMR $\left(500 \mathrm{MHz}, \mathrm{CDCl}_{3}\right) \delta$ 7.22-7.03 (m, 5H), 6.80 (s, 1H), 6.00 (br s, 1H), 5.83 (br s, 1H), $3.00(\mathrm{AB} \mathrm{q}, J=13.7 \mathrm{~Hz}, 2 \mathrm{H}), 2.23-1.96(\mathrm{~m}, 6 \mathrm{H}), 0.89$ (t, $J=7.5 \mathrm{~Hz}, 3 \mathrm{H}) ;{ }^{13} \mathrm{C} \mathrm{NMR}\left(125 \mathrm{MHz}, \mathrm{CDCl}_{3}\right) \delta 174.7,173.3,149.9,136.5,134.5,130.4,128.3$, 127.1, 88.1, 44.5, 31.5, 29.1, 18.4, 11.8; IR 3346, 1747, $1669 \mathrm{~cm}^{-1}$; CIMS $274\left(\mathrm{M}^{+}+1,100\right)$. Anal. Calcd for $\mathrm{C}_{16} \mathrm{H}_{19} \mathrm{NO}_{3}$ : C, 70.31; H, 7.01; N, 5.12. Found: C, 70.48; H, 7.03; N, 5.07.

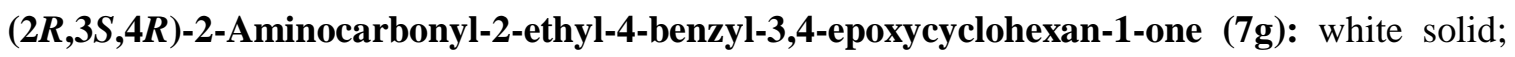
mp $109-11{ }^{\circ} \mathrm{C} ;[\alpha]^{25}{ }_{\mathrm{D}}-46.4\left(c 1.2, \mathrm{CHCl}_{3}\right) ;{ }^{1} \mathrm{H}$ NMR $\left(500 \mathrm{MHz}, \mathrm{CDCl}_{3}\right) \delta$ 7.32-7.23 (m, 5H), 
6.87 (br s, 1H), 5.89 (br s, 1H), $3.51(\mathrm{~s}, 1 \mathrm{H}), 3.00(\mathrm{AB} \mathrm{q}, J=14.2 \mathrm{~Hz}, 2 \mathrm{H}), 2.48(\mathrm{~m}, 1 \mathrm{H}), 2.20$ (m, 2H), $2.05(\mathrm{~m}, 2 \mathrm{H}), 1.75(\mathrm{~m}, 2 \mathrm{H}), 0.80(\mathrm{t}, J=7.5 \mathrm{~Hz}, 3 \mathrm{H}) ;{ }^{13} \mathrm{C} \mathrm{NMR}\left(125 \mathrm{MHz}, \mathrm{CDCl}_{3}\right) \delta$ 209.3, $171.1,136.1,129.8,128.8,127.3,61.0,60.9,59.5,42.6,35.6,29.5,24.4,9.4$; IR 3345, 1682, $1462 \mathrm{~cm}^{-1}$; CIMS $274\left(\mathrm{M}^{+}+1,100\right)$. Anal. Calcd for $\mathrm{C}_{16} \mathrm{H}_{19} \mathrm{NO}_{3}: \mathrm{C}, 70.31 ; \mathrm{H}, 7.01 ; \mathrm{N}, 5.12$. Found: C, 70.19; H, 6.96; N, 5.08.

Cyclization of $\mathbf{5 f}$. To a solution of butenolide amide $\mathbf{5 f}(200 \mathrm{mg}, 1.01 \mathrm{mmol})$ and $\mathrm{Si}(\mathrm{OEt})_{4}(0.49$ $\mathrm{mL}, 2.20 \mathrm{mmol})$ in $\mathrm{CH}_{2} \mathrm{Cl}_{2}(2 \mathrm{~mL})$ was added $\mathrm{CsF}(334 \mathrm{mg}, 2.20 \mathrm{mmol})$ at room temperature. The reaction mixture was stirred overnight and diluted with water. The reaction mixture was then extracted with $10 \% \mathrm{MeOH}$ in $\mathrm{CH}_{2} \mathrm{Cl}_{2}$. The combined organic layers were dried over anhydrous $\mathrm{Na}_{2} \mathrm{SO}_{4}$ and concentrated under reduced pressure. Flash column chromatography (EtOAc) of the crude residue gave an inseparable mixture of $\mathbf{1 4}$ and $\mathbf{1 5}$ in a ratio of 1:1 (152 $\mathrm{mg}, 76 \%)$ as a white solid. $\quad(3 R, 3 a R, 7 a R)-3$-Ethyltertahydro-7a-methyl-furo[3,2-b]pyridine-2,5(3H,4H)dione (14); white solid; ${ }^{1} \mathrm{H}$ NMR (500 MHz, $\left.\mathrm{CDCl}_{3}\right) \delta 7.53$ (br s, $\left.1 \mathrm{H}\right), 3.60$ (dd, $J=6.8,3.4 \mathrm{~Hz}$, 1H), $2.60(\mathrm{~m}, 1 \mathrm{H}), 2.47-1.70(\mathrm{~m}, 6 \mathrm{H}), 1.57(\mathrm{~s}, 3 \mathrm{H}), 1.09(\mathrm{t}, J=7.5 \mathrm{~Hz}, 3 \mathrm{H}) ;{ }^{13} \mathrm{C} \mathrm{NMR}(125 \mathrm{MHz}$, $\left.\mathrm{CDCl}_{3}\right) \delta 175.7,172.1,79.4,61.2,51.6,30.9,27.9,26.3,23.1,11.4 ;$ IR 1769, $1662 \mathrm{~cm}^{-1}$; CIMS

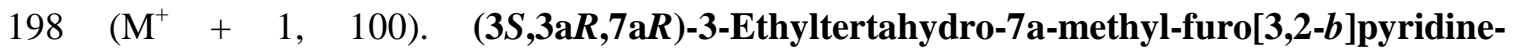
2,5(3H,4H)-dione (15). The same reaction described above was performed for $72 \mathrm{~h}$. Flash column chromatography (EtOAc) of the crude residue provided $\mathbf{1 5}(122 \mathrm{mg}, 62 \%)$ as a white solid. The compound was crystallized from hexanes/EtOAc $(3: 1) .[\alpha]_{D}^{25}-116.7\left(c 1.2, \mathrm{CHCl}_{3}\right)$; ${ }^{1} \mathrm{H}$ NMR (500 MHz, $\mathrm{CDCl}_{3}$ ) $\delta 6.94$ (br s, 1H), $3.93(\mathrm{~d}, J=6.1 \mathrm{~Hz}, 1 \mathrm{H}), 2.76(\mathrm{~m}, 1 \mathrm{H}), 2.48-1.52$ (m, 6H), $1.50(\mathrm{~s}, 3 \mathrm{H}), 1.06(\mathrm{t}, J=7.5 \mathrm{~Hz}, 3 \mathrm{H}) ;{ }^{13} \mathrm{C} \mathrm{NMR}\left(125 \mathrm{MHz}, \mathrm{CDCl}_{3}\right) \delta 176.1,172.0,79.4$, 58.7, 47.3, 31.1, 26.4, 25.5, 17.9, 12.4; IR 1769, $1662 \mathrm{~cm}^{-1}$; CIMS $198\left(\mathrm{M}^{+}+1\right.$, 100). Anal. Calcd for $\mathrm{C}_{10} \mathrm{H}_{15} \mathrm{NO}_{3}: \mathrm{C}, 60.90 ; \mathrm{H}, 7.67 ; \mathrm{N}, 7.10$. Found: C, 60.68; H, 7.63; N, 6.93. 
Hoffmann Rearrangement of 5f. To a solution of butenolide amide $\mathbf{5 f}(160 \mathrm{mg}, 0.80 \mathrm{mmol})$ in pyridine $(8 \mathrm{~mL})$ was added $\mathrm{Pb}(\mathrm{OAc})_{4}(1.06 \mathrm{~g}, 2.40 \mathrm{mmol})$ at room temperature. The reaction mixture was stirred at $60{ }^{\circ} \mathrm{C}$ for $2 \mathrm{~h}$. The resulting mixture was then treated with anhydrous $t$ $\mathrm{BuOH}(600 \mu \mathrm{L})$ via syringe and stirred at $60{ }^{\circ} \mathrm{C}$ overnight. The reaction mixture was cooled to room temperature and diluted with diethyl ether and washed with water and brine. The combined organic layers were dried over anhydrous $\mathrm{Na}_{2} \mathrm{SO}_{4}$ and concentrated under reduced pressure. Flash column chromatography (hexanes/EtOAc, 2:1) of the crude residue gave $\mathbf{1 6}$ (192 $\mathrm{mg}, 88 \%$ ) as a pale yellow oil. (5R)-[3-Ethyl-5-methyl-5-[[2'-(t-butoxy)carbonylamino]ethyl]furan-2(5H)one (16): $[\alpha]^{25}-25.7\left(c\right.$ 1.4, $\left.\mathrm{CHCl}_{3}\right) ;{ }^{1} \mathrm{H} \mathrm{NMR}\left(500 \mathrm{MHz}, \mathrm{CDCl}_{3}\right) \delta 6.91$ (s, $\left.1 \mathrm{H}\right), 4.76$ (br s, 1 H), 3.04 (m, 2 H), $2.22(\mathrm{AB} \mathrm{q}, J=7.4 \mathrm{~Hz}, 2 \mathrm{H}), 1.90$ (m, $2 \mathrm{H}), 1.38(\mathrm{~s}, 3 \mathrm{H}), 1.36$ (s, $6 \mathrm{H}), 1.09$ (t, $J=7.4 \mathrm{~Hz}, 3 \mathrm{H}) ;{ }^{13} \mathrm{C}$ NMR $\left(125 \mathrm{MHz}, \mathrm{CDCl}_{3}\right) \delta 173.0,155.9,151.3,135.1,85.7,79.3,38.3$, 36.0, 28.4, 24.4, 18.5, 11.8; IR 3855, 1756, $1704 \mathrm{~cm}^{-1}$; CIMS $270\left(\mathrm{M}^{+}+1,2\right), 214$ (100). Anal. Calcd for $\mathrm{C}_{14} \mathrm{H}_{23} \mathrm{NO}_{4}: \mathrm{C}, 62.43 ; \mathrm{H}, 8.61 ; \mathrm{N}, 5.20$. Found: C, 62.22; H, 8.61; N, 5.14.

Cyclization of 16 in the Presence of TFA. To a solution of 16 (168 mg, $0.62 \mathrm{mmol})$ in $\mathrm{CH}_{2} \mathrm{Cl}_{2}$ $(10 \mathrm{~mL})$ was added TFA $(0.5 \mathrm{~mL})$ at room temperature. The reaction mixture was stirred overnight and neutralized with saturated aqueous $\mathrm{NaHCO}_{3}$ solution and extracted with $\mathrm{CH}_{2} \mathrm{Cl}_{2}$. The combined organic layers were dried over anhydrous $\mathrm{Na}_{2} \mathrm{SO}_{4}$ and concentrated under reduced pressure. Flash column chromatography $(\mathrm{MeOH} / \mathrm{EtOAc}, 1: 10)$ of the crude residue gave a mixture of $\mathbf{1 8}$ and $\mathbf{1 9}(63 \mathrm{mg}, 95 \%)$ in a ratio of $1: 7$ as a yellow oil. (3R,3aR,6aR)-3Ethylhexahydro-6a-methyl-2H-furo[3,2-b]pyrrol-2-one (18): yellow oil; $[\alpha]^{25}{ }_{D}+37.5(c 0.4$, $\left.\mathrm{CHCl}_{3}\right) ;{ }^{1} \mathrm{H} \mathrm{NMR}\left(500 \mathrm{MHz}, \mathrm{CDCl}_{3}\right) \delta 3.54(\mathrm{~d}, J=6.6 \mathrm{~Hz}, 1 \mathrm{H}), 3.00(\mathrm{~m}, 2 \mathrm{H}), 2.54(\mathrm{~m}, 1 \mathrm{H}), 2.19$ $(\mathrm{m}, 1 \mathrm{H}), 1.85(\mathrm{~m}, 3 \mathrm{H}), 1.60(\mathrm{~m}, 1 \mathrm{H}), 1.50(\mathrm{~s}, 3 \mathrm{H}), 1.03(\mathrm{t}, J=7.3 \mathrm{~Hz}, 3 \mathrm{H}) ;{ }^{13} \mathrm{C}$ NMR $(125 \mathrm{MHz}$, $\left.\mathrm{CDCl}_{3}\right) \delta 178.0,92.0,65.0,46.3,45.7,40.0,23.1,18.7,12.6 ;$ IR $3853,1759 \mathrm{~cm}^{-1} ; \mathrm{CIMS} 170\left(\mathrm{M}^{+}\right.$ 
$+1,100)$; HRMS calcd for $\mathrm{C}_{9} \mathrm{H}_{15} \mathrm{NO}_{2}\left(\mathrm{M}^{+}+1\right)$ 170.1182, found 170.1179 . (3S,3aR,6aR)-3Ethylhexahydro-6a-methyl-2H-furo[3,2-b]pyrrol-2-one (19): yellow oil; $[\alpha]_{D}^{25}+39.1(c$ 1.1, $\left.\mathrm{CHCl}_{3}\right) ;{ }^{1} \mathrm{H}$ NMR (500 MHz, $\left.\mathrm{CDCl}_{3}\right) \delta 3.34(\mathrm{~d}, J=2.5 \mathrm{~Hz}, 1 \mathrm{H}), 3.00(\mathrm{~m}, 2 \mathrm{H}), 2.40-1.62(\mathrm{~m}, 6 \mathrm{H})$, $1.56(\mathrm{~s}, 3 \mathrm{H}), 1.06(\mathrm{t}, J=7.5 \mathrm{~Hz}, 3 \mathrm{H}) ;{ }^{13} \mathrm{C} \mathrm{NMR}\left(125 \mathrm{MHz}, \mathrm{CDCl}_{3}\right) \delta 178.8,92.5,68.6,51.4$, 45.5, 40.8, 25.7, 24.6, 11.9; IR $1755 \mathrm{~cm}^{-1}$; CIMS $170\left(\mathrm{M}^{+}+1,100\right)$; HRMS calcd for $\mathrm{C}_{9} \mathrm{H}_{15} \mathrm{NO}_{2}$ $\left(\mathrm{M}^{+}+1\right)$ 170.1182, found 170.1178 . 


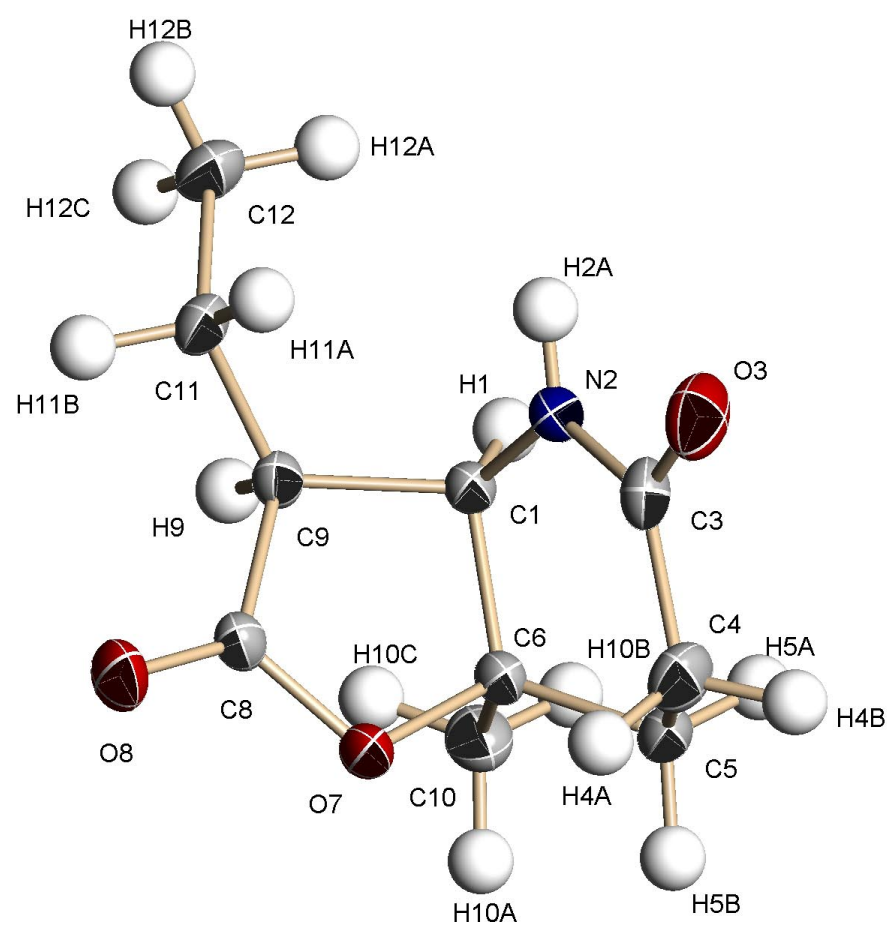

Figure 1. ORTEP drawing of 15. 


\section{Table 1. Crystal data and structure refinement for 15.}

\begin{tabular}{|c|c|c|}
\hline Identification code & \multicolumn{2}{|l|}{15 (rpi21m) } \\
\hline Empirical formula & \multicolumn{2}{|l|}{ C10 H15 N O3 } \\
\hline Formula weight & \multicolumn{2}{|l|}{197.23} \\
\hline Temperature & \multicolumn{2}{|l|}{ 198(2) K } \\
\hline Wavelength & \multicolumn{2}{|l|}{$0.71073 \AA$} \\
\hline Crystal system & \multicolumn{2}{|l|}{ Orthorhombic } \\
\hline Space group & \multicolumn{2}{|l|}{$\mathrm{C} 222(1)$} \\
\hline \multirow[t]{3}{*}{ Unit cell dimensions } & $\mathrm{a}=10.6675(4) \AA$ & $\alpha=90^{\circ}$ \\
\hline & $\mathrm{b}=11.0586(5) \AA$ & $\beta=90^{\circ}$. \\
\hline & $c=16.5092(7) \AA$ & $\gamma=90^{\circ}$ \\
\hline Volume & \multicolumn{2}{|l|}{$1947.55(14) \AA^{3}$} \\
\hline $\mathrm{Z}$ & \multicolumn{2}{|l|}{8} \\
\hline Density (calculated) & \multicolumn{2}{|l|}{$1.345 \mathrm{Mg} / \mathrm{m}^{3}$} \\
\hline Absorption coefficient & \multicolumn{2}{|l|}{$0.099 \mathrm{~mm}^{-1}$} \\
\hline $\mathrm{F}(000)$ & \multicolumn{2}{|l|}{848} \\
\hline Crystal size & \multicolumn{2}{|c|}{$0.56 \times 0.40 \times 0.39 \mathrm{~mm}^{3}$} \\
\hline Theta range for data collection & \multicolumn{2}{|l|}{2.47 to $28.27^{\circ}$. } \\
\hline Index ranges & \multicolumn{2}{|c|}{$-14<=\mathrm{h}<=10,-14<=\mathrm{k}<=14,-22<=\mathrm{l}<=21$} \\
\hline Reflections collected & \multicolumn{2}{|l|}{7904} \\
\hline Independent reflections & \multicolumn{2}{|c|}{$2420[\mathrm{R}(\mathrm{int})=0.0189]$} \\
\hline Completeness to theta $=28.27^{\circ}$ & \multicolumn{2}{|l|}{$99.9 \%$} \\
\hline Absorption correction & \multicolumn{2}{|l|}{ SADABS } \\
\hline Max. and min. transmission & \multicolumn{2}{|l|}{0.9623 and 0.9465} \\
\hline Refinement method & \multicolumn{2}{|c|}{ Full-matrix least-squares on $\mathrm{F}^{2}$} \\
\hline Data / restraints / parameters & \multicolumn{2}{|l|}{$2420 / 0 / 147$} \\
\hline Goodness-of-fit on $\mathrm{F}^{2}$ & \multicolumn{2}{|l|}{1.034} \\
\hline Final R indices $[\mathrm{I}>2 \operatorname{sigma}(\mathrm{I})]$ & \multicolumn{2}{|c|}{$\mathrm{R} 1=0.0303, \mathrm{wR} 2=0.0810$} \\
\hline $\mathrm{R}$ indices (all data) & \multicolumn{2}{|c|}{$\mathrm{R} 1=0.0312, \mathrm{wR} 2=0.0822$} \\
\hline Absolute structure parameter & \multicolumn{2}{|l|}{$0.3(7)$} \\
\hline Largest diff. peak and hole & \multicolumn{2}{|c|}{0.269 and -0.151 e. $\AA^{-3}$} \\
\hline
\end{tabular}


$14(\mathrm{E}=17.0689 \mathrm{kcal} / \mathrm{mol})$

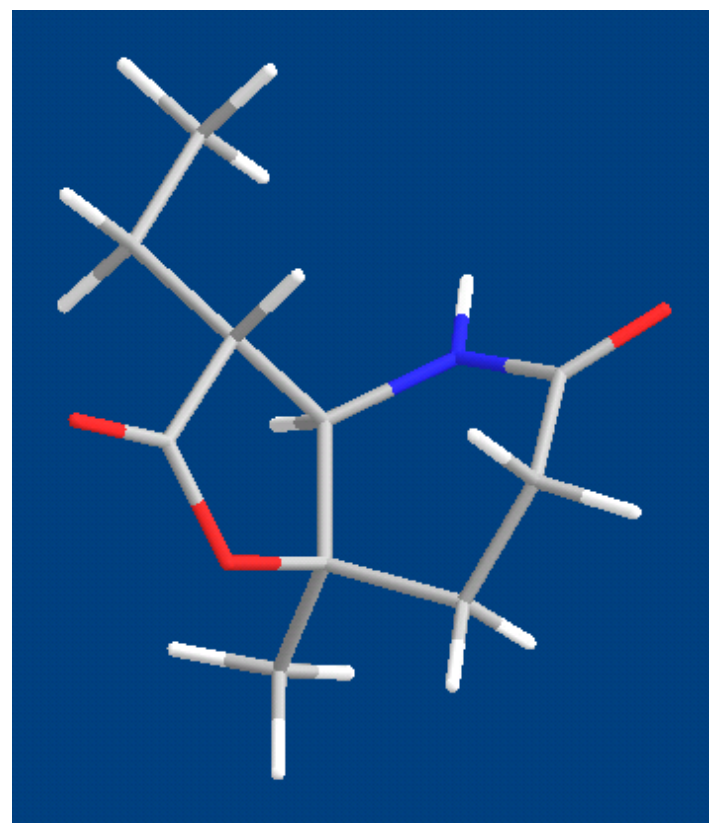

$15(\mathrm{E}=16.7884 \mathrm{kcal} / \mathrm{mol})$

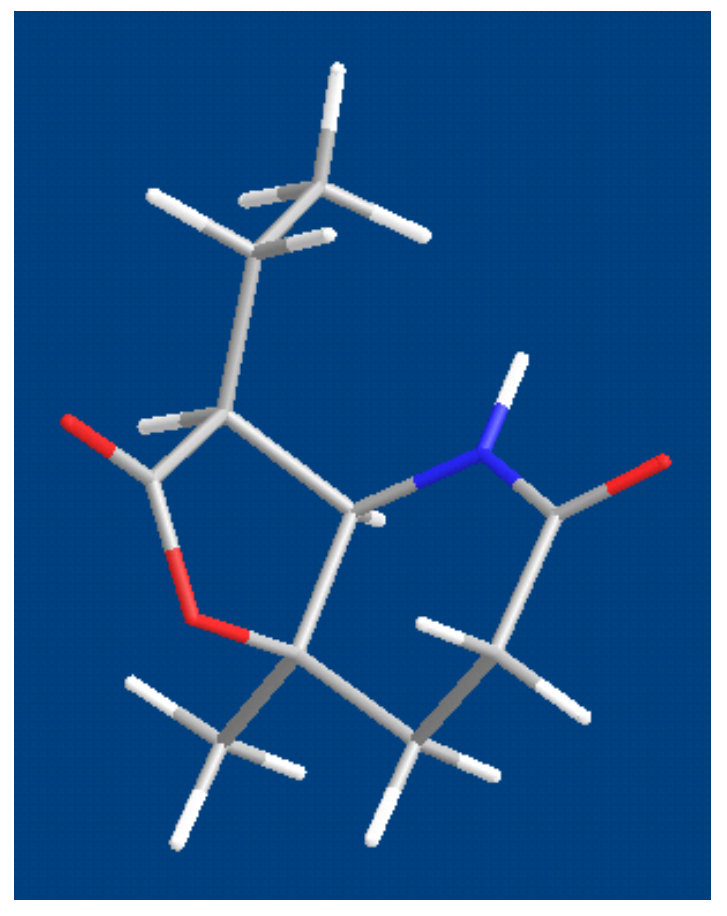

Figure 2. Energy-minimized structures of $\mathbf{1 4}$ and $\mathbf{1 5 .}$ 
$18(\mathrm{E}=18.5587 \mathrm{kcal} / \mathrm{mol})$

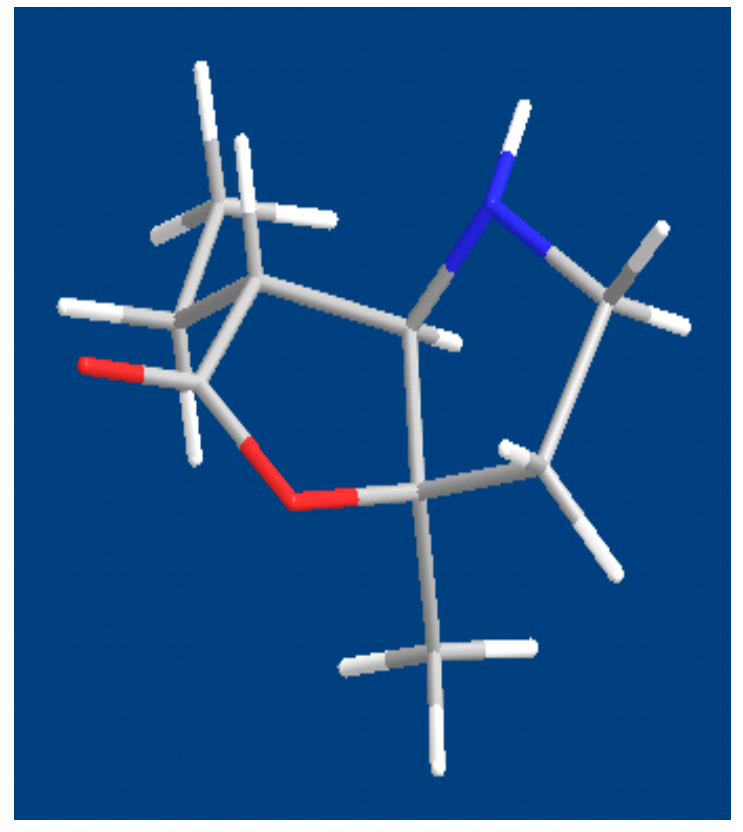

$19(\mathrm{E}=17.6032 \mathrm{kcal} / \mathrm{mol})$

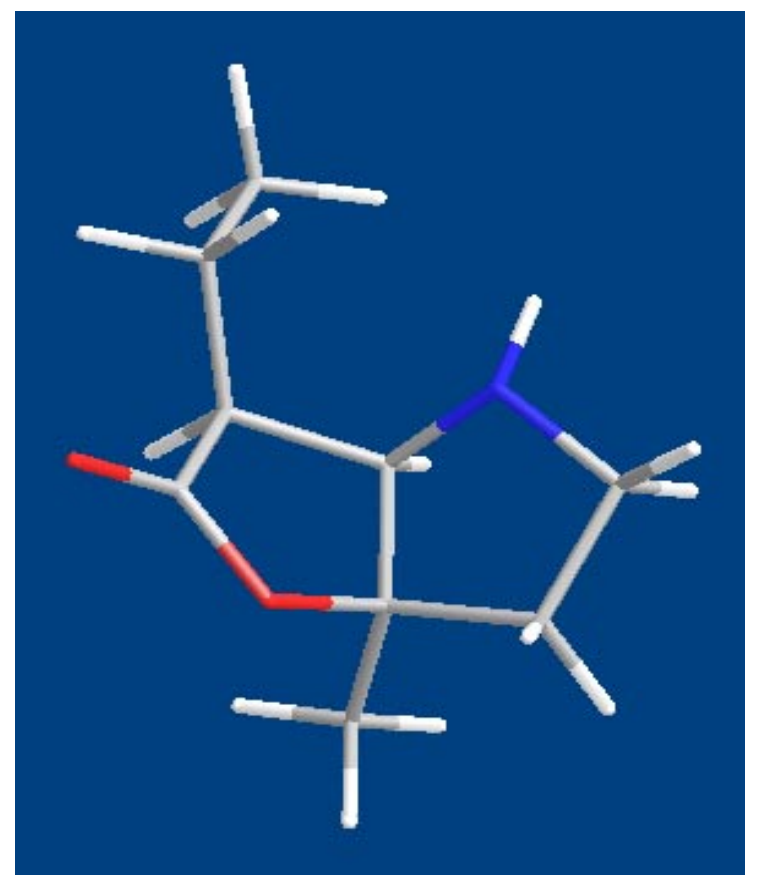

Figure 3. Energy-minimized structures of $\mathbf{1 8}$ and 19. 


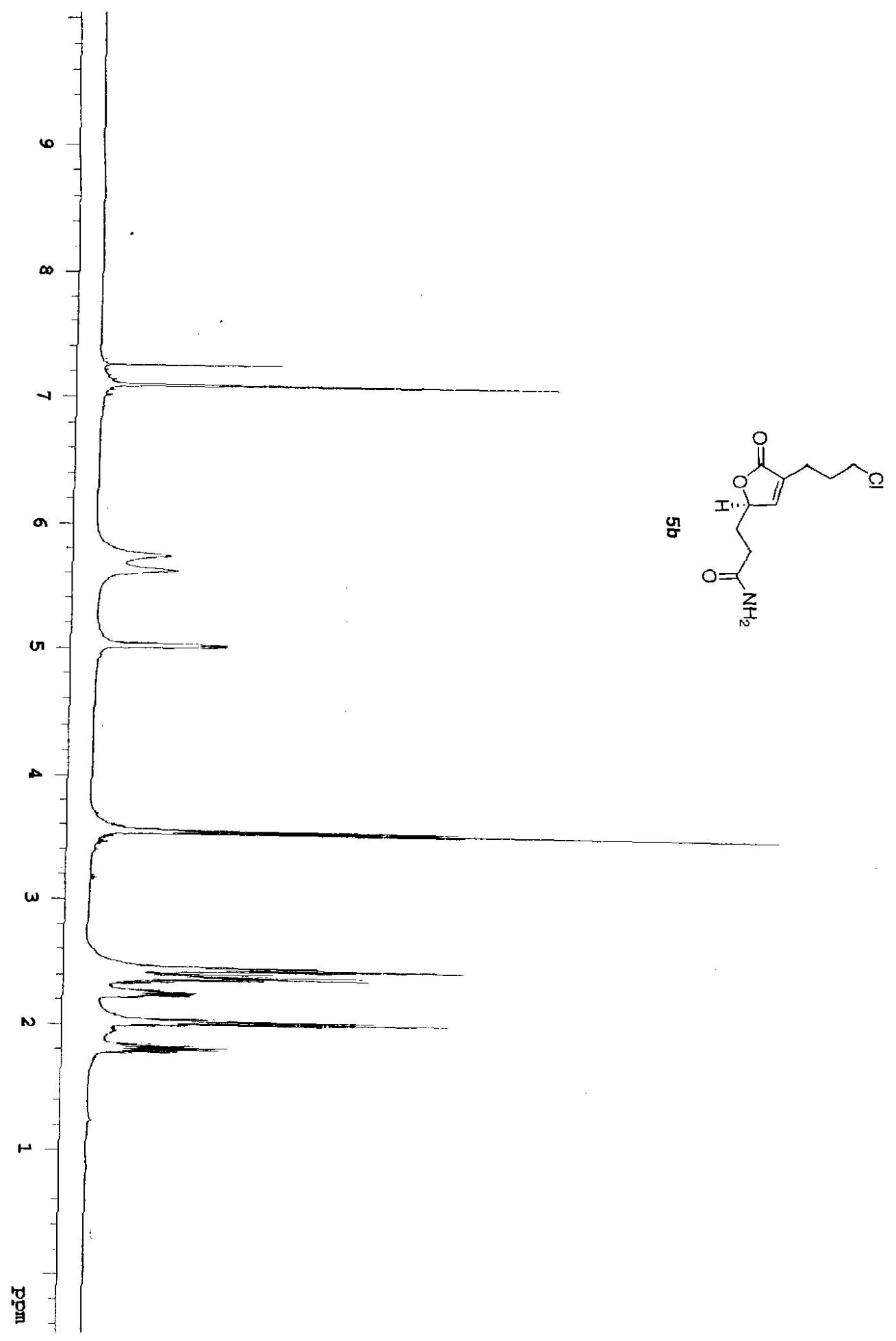




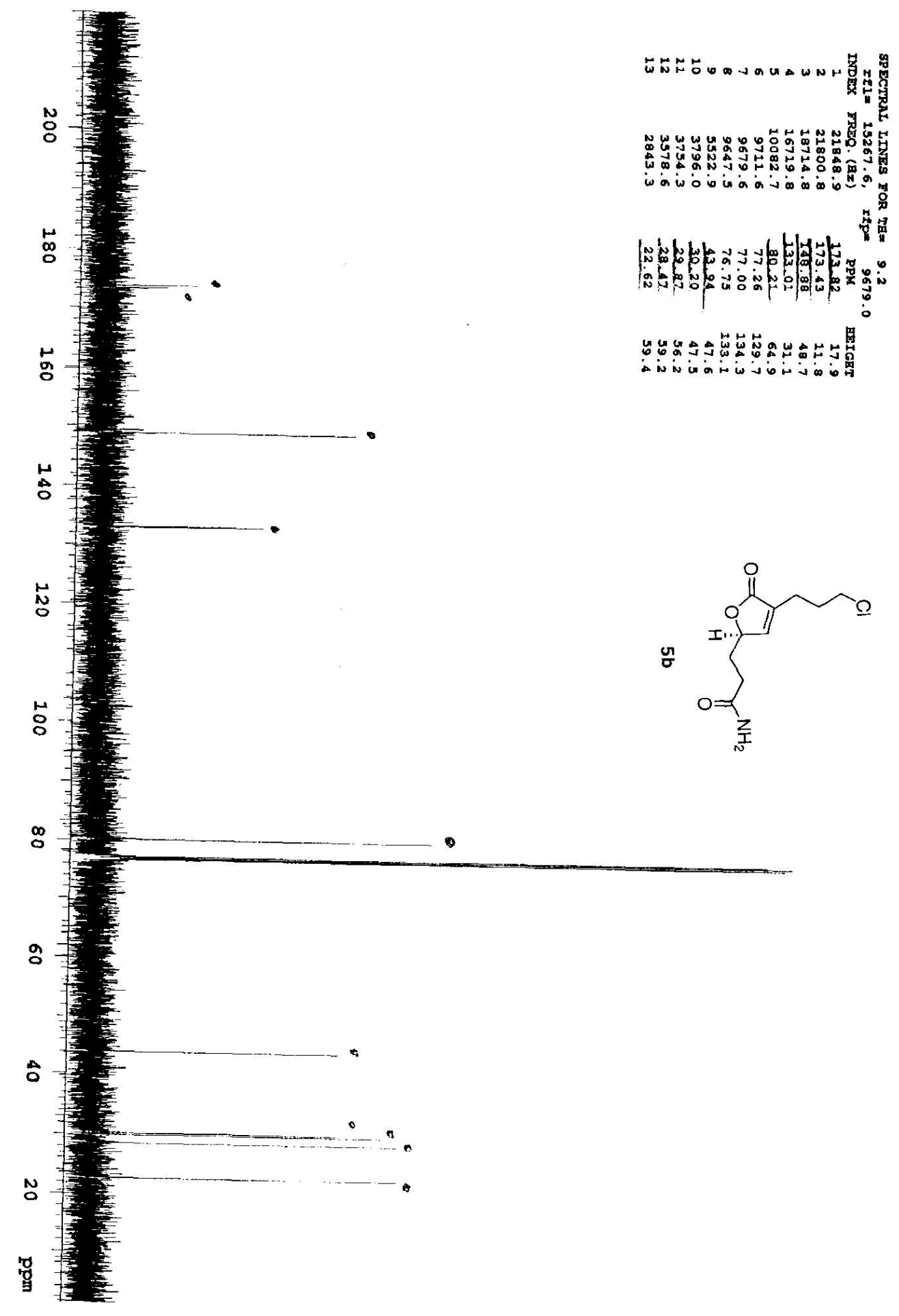




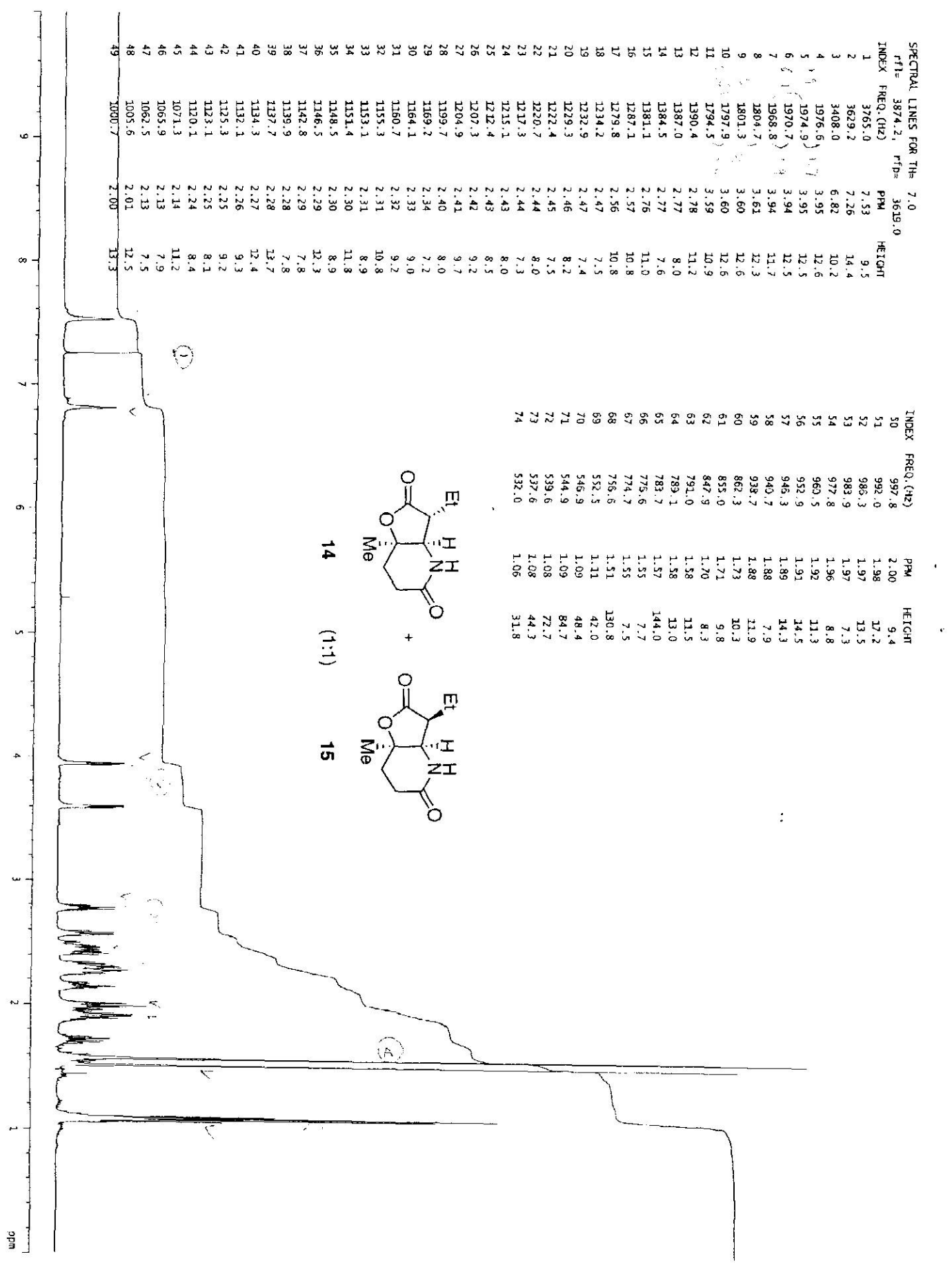



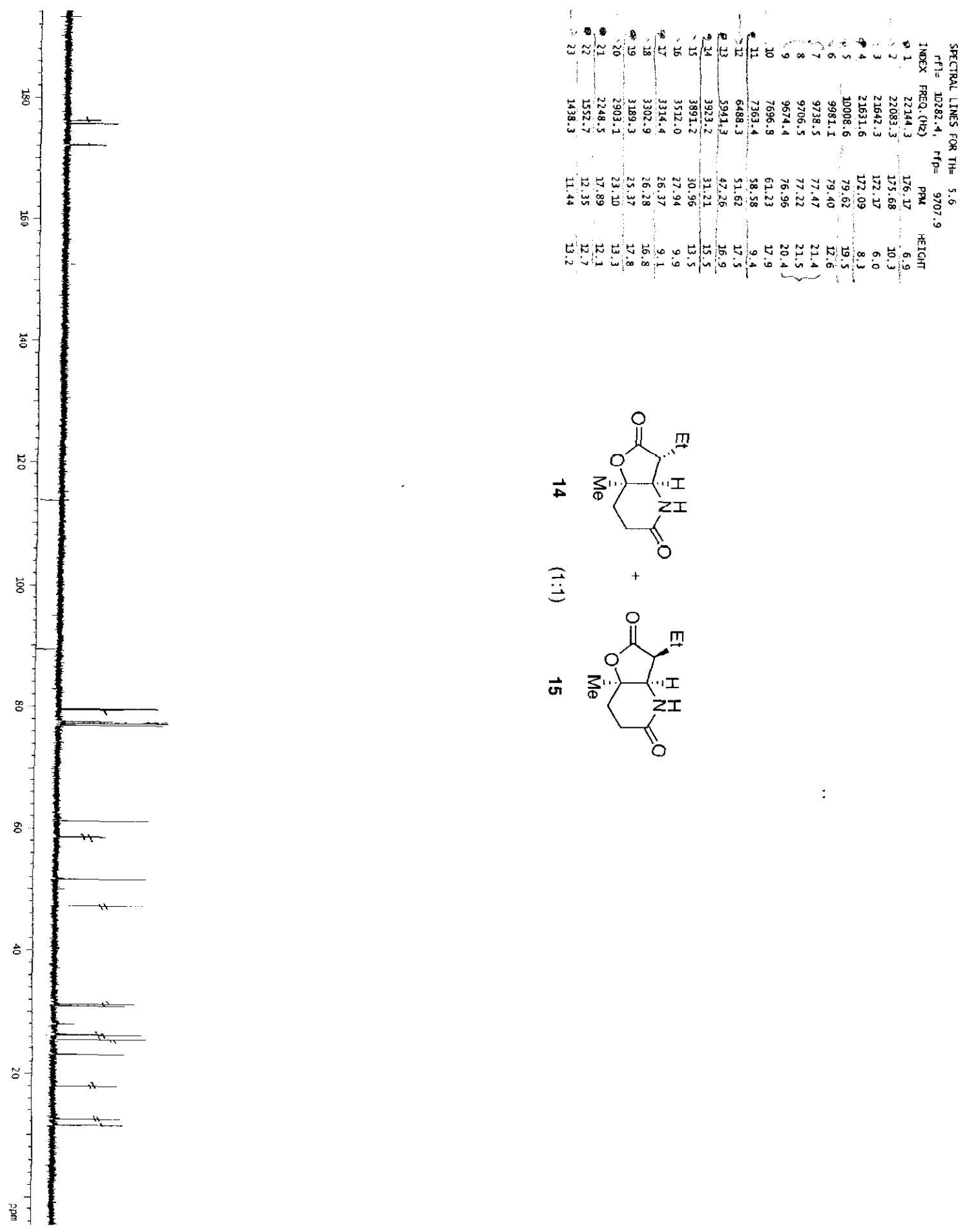


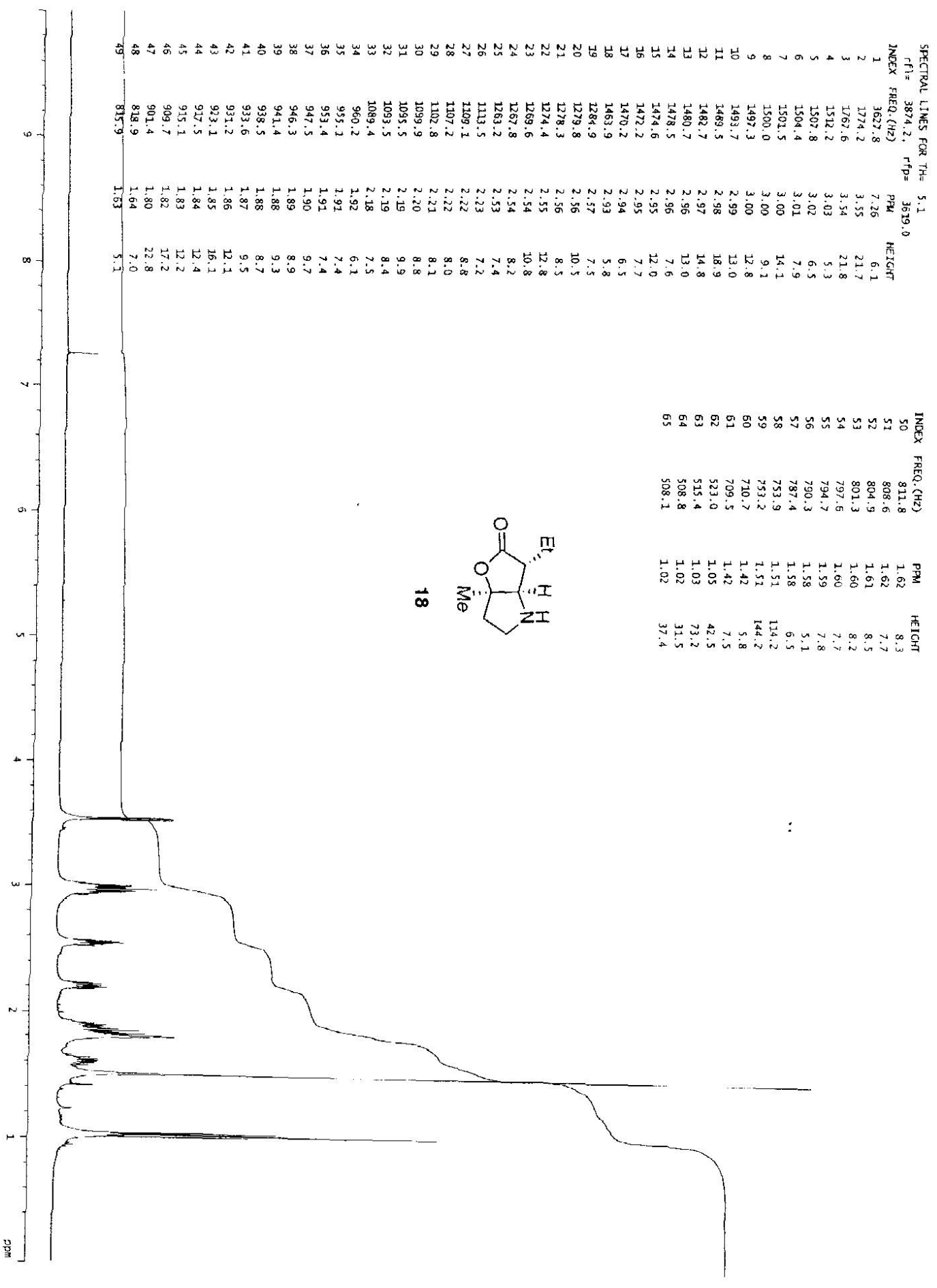




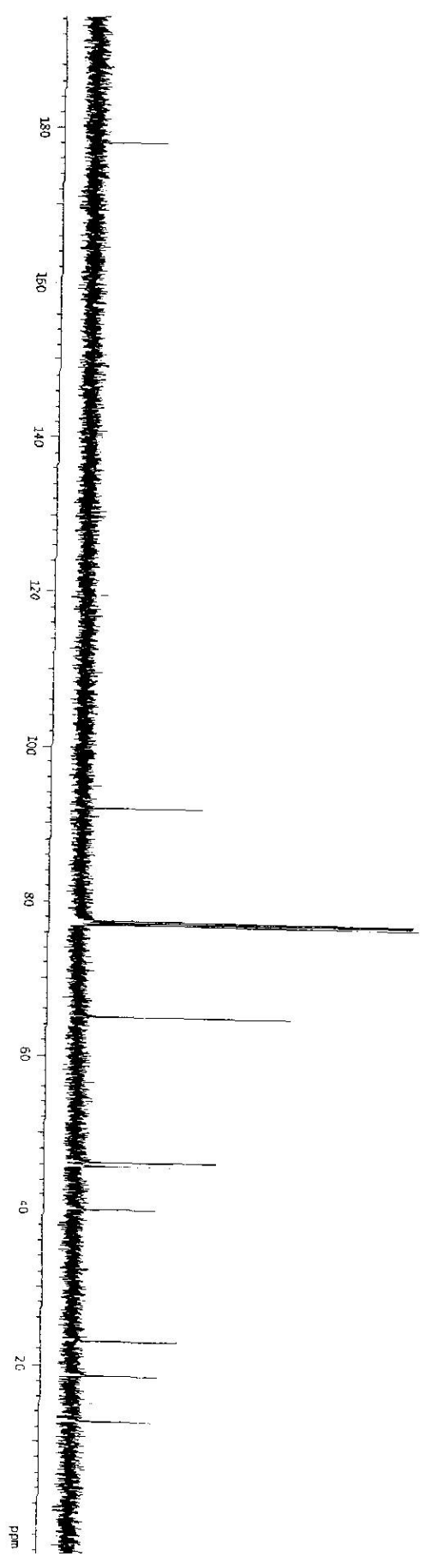

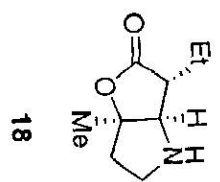

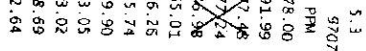

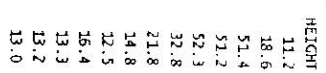

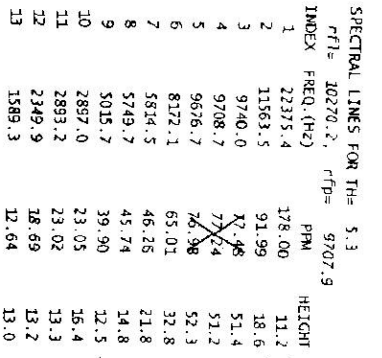




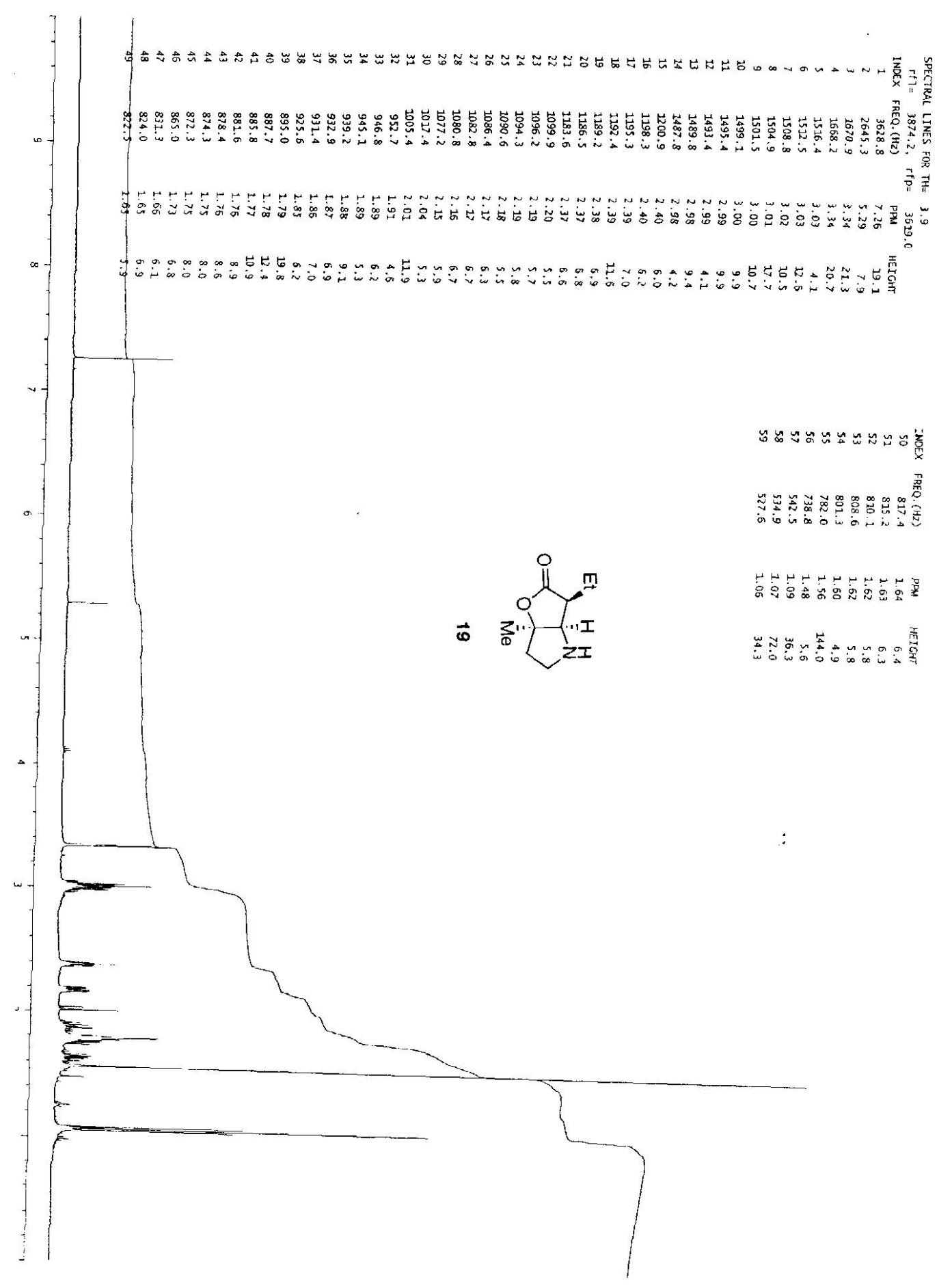




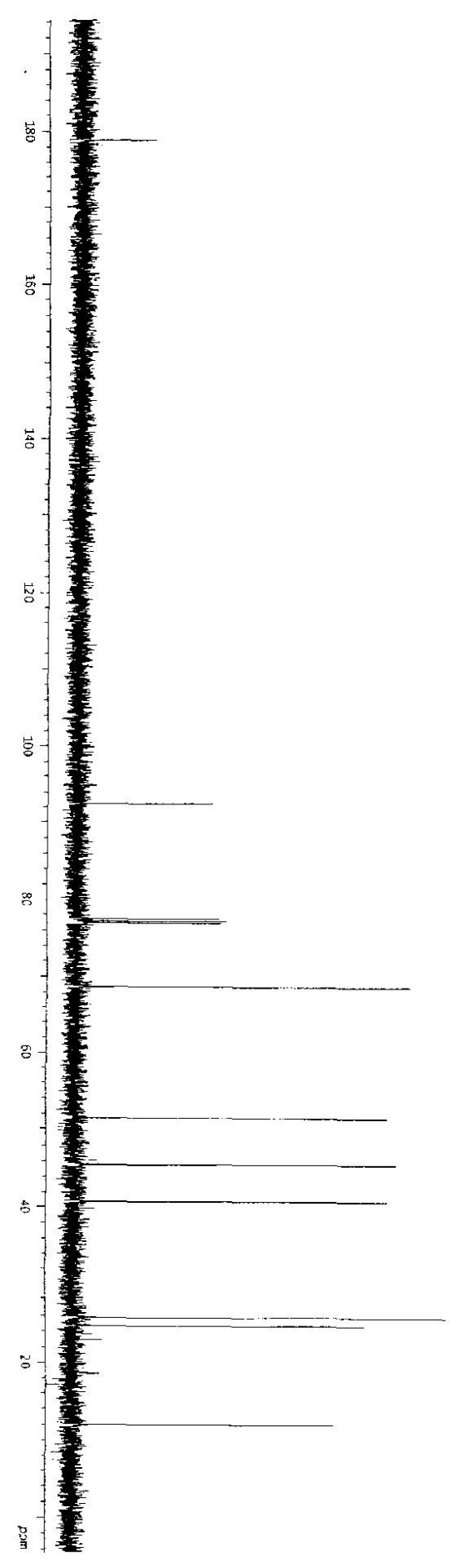

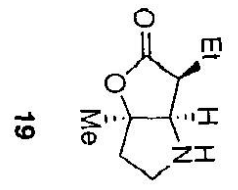

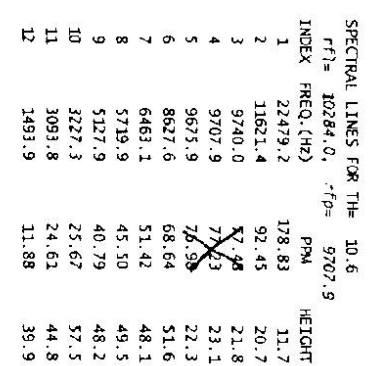

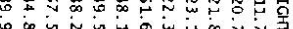

